\title{
LEVANTAMENTO SOBRE AS AÇÕES DE ENFERMAGEM NO PROGRAMA DE CONTROLE DA HANSENÍASE NO ESTADO DE SÃO PAULO*
}

Elisete Silva Pedrazzani**

Discute-se sobre as ações de enfermagem no Programa de Controle da Hanseníase no Estado de São Paulo destacando como sendo primordiais as ações assistenciais, educativas, de vigilância epidemiológica e administrativa, ressalta-se ainda a necessidade de revisão das práticas desenvolvidas no novo modelo de assistência à saúde.

UNITERMOS: enfermagem, prática, hanseníase

\section{INTRODUÇÃO}

A realização da $1^{\mathrm{a}}$ Oficina de Trabalho sobre Ações de Enfermagem em Hanseníase pela Coordenação do Programa de Controle da Secretaria de Estado da Saúde de São Paulo, no ano de 1991, permitiu que um grupo assessor formado por enfermeiros, organizasse o referido evento que teve como objetivo geral avaliar a situação das ações de enfermagem em hanseníase nas unidades de saúde que compõem o sistema de saúde da Secretaria de Estado da Saúde de São Paulo, assim como, identificar estratégias de intervenção adequadas à assistência integral aos portadores de hanseníase no atual modelo assistencial ${ }^{4}$.

\footnotetext{
*Apresentado na $1^{a}$ Oficina de Ações de Enfermagem em Hanseníase

**Professor Doutor do Departamento de Enfermagem da Universidade Federal de São Carlos
} 
As discussões para elaboração do programa final da Oficina com a finalidade de aprofundar o debate nos grupos apontavam em duas direções: a primeira relacionada aos problemas inerentes à própria profissão no que diz respeito ao distanciamento entre o ensino e a prática, ou seja, a questão da divisão técnica do trabalho na enfermagem e o baixo reconhecimento do potencial de trabalho do enfermeiro; a Segunda direção apontava para questões mais diretamente relacionadas à problemática de controle da hanseníase, ou seja, a não priorização por parte dos órgãos públicos das ações do Programa de Controle da Hanseníase, alta rotatividade de profissionais na área, baixa capacitação técnica para o trabalho na área apesar do alto número de treinamentos realizados e ainda o baixo interesse dos profissionais no trabalho junto à área.

O objetivo da apresentação desses dados foi ode fornecer subsídios ao grupo de enfermeiros presentes na Oficina de Trabalho visando contribuir na avaliação da situação das ações de enfermagem em hanseníase assim como identificar estratégias de intervenção que pudessem estimular e instrumentalizar os enfermeiros no desempenho de suas funções.

\section{A CARACTERIZAÇÃO DAS AÇÕES DE ENFERMAGEM}

Em reunião patrocinada pela Organização Pan-americana da Saúde, realizada no segundo semestre de 1989, no Rio de Janeiro, reunindo enfermeiros do Brasil, Argentina e da própria Organização discutiu-se a participação do pessoal de enfermagem no controle da hanseníase.

Objetivando a melhoria da prática de enfermagem o a implementação de conhecimentos disponíveis sobre o controle da doença nos serviços locais de saúde da região, tomou-se como referência a participação do pessoal de enfermagem em sete áreas básicas: prevenção da hanseníase, busca e diagnóstico dos casos, tratamento e seguimento dos pacientes, prevenção e tratamento de incapacidade, gerência das atividades de controle, sistema de registro e vigilância epidemiológica e pesquisas.

$\mathrm{Na}$ primeira área, prevenção da hanseníase uma das tarefas fundamentais objetivando a promoção da saúde e prevenção da doença, é a da participação social, que inclui dentre outras, a capacitação do pessoal de enfermagem e a conscientização da população como o meio mais adequado para o desenvolvimento da auto-responsabilidade e autocuidado do paciente e família.

$\mathrm{Na}$ busca e diagnóstico dos casos devem ser desempenhadas tarefas relevantes que, dentro dos princípios da integralidade no atendimento, contribuem para que o enfermeiro consiga estabelecer uma relação efetiva com os usuários.

Em relação ao tratamento e seguimento de pacientes destaca-se a necessidade de uma atitude crítica, que permita uni aprimoramento no seu saber diário, com incorporação de todo o conhecimento técnico-científico que vem sendo adotado nessa área, como por exemplo, a multidrogaterapia. A prevenção e o tratamento de incapacidades merecem destaques especialmente no acompanhamento do paciente com reações hansênicas e conseqüente atuação nos casos que necessitem de prescrição e/ou execução de técnicas de prevenção e tratamento de incapacidades tanto para mãos, pés, olhos e nariz.

$\mathrm{Na}$ área administrativa, o enfermeiro tem desempenhado sua atuação em dois 
setores distintos: gerência da assistência de enfermagem propriamente dita e a gerência e/ou coordenação de programas de controle.

Quanto à vigilância epidemiológica, o sistema de registro é de fundamental importância na avaliação epidemiológica e operacional das atividades de controle da hanseníase, em qualquer nível de atuação desde a unidade básica que se constitui na porta de entrada do sistema, até o nível central.

A pesquisa surge como um instrumento preciso na produção de novos conhecimentos, que permitem um aprimoramento da atuação profissional e também a análise critica dos problemas inerentes à profissão e à doença propriamente dita.

Em todas essas áreas de atuação do enfermeiro, entendemos que o trabalho deve se dar de forma integrada com os demais membros da equipe multiprofissional da unidade de saúde, respeitando-se um princípio básico da integralidade da assistência à saúde do indivíduo.

Em estudo 6 realizado junto a docentes dos Cursos de Graduação em Enfermagem do Estado de São Paulo que ministram o tema hanseníase e aos enfermeiros das unidades de saúde que atuam junto ao Programa de Controle da Hanseníase em diferentes níveis, cujo objetivo era o de aliviar a situação do ensino e da prática do enfermeiro em hanseníase, foi destacado dentre outros aspectos importantes da atuação desse profissional segundo o ponto de vista dos docentes (tabela 1) e dos enfermeiros (tabela 2), as ações relacionadas à assistência de enfermagem e educação em saúde.

\section{TABELA 1 - DISTRIBUIÇÃO DAS RESPOSTAS DOS DOCENTES SOBRE OS ASPECTOS IMPORTANTES DA ATUAÇÃO DO ENFERMEIRO EM HANSENÍASE}

\begin{tabular}{|l|r|r|}
\hline \multirow{2}{*}{ Aspectos importantes } & \multicolumn{2}{|c|}{ Respostas } \\
\cline { 2 - 3 } & $\mathbf{N .}^{\mathbf{0}}$ & \multicolumn{1}{c|}{$\%$} \\
\hline Assistência de enfermagem & & \\
$\quad$ - prevenção de incapacidades & 10 & 24,39 \\
- controle de tratamento & 4 & 9,75 \\
- consulta de enfermagem & 3 & 7,32 \\
- controle clínico & 1 & 2,44 \\
- coleta de material para baciloscopia & 1 & 2,44 \\
- teste de Mitsuda & 1 & 2,44 \\
\hline Subtotal & $\mathbf{2 0}$ & $\mathbf{4 8 , 7 8}$ \\
\hline Educação em saúde & & \\
- prevenção da doença & 2 & 4,88 \\
- trabalho de grupo & 1 & 2,44 \\
- sem especificar & 13 & 31,70 \\
\hline Subtotal & $\mathbf{1 6}$ & $\mathbf{3 9 , 0 2}$ \\
\hline Vigilância epidemiológica & & \\
- vista domiciliária & 2 & 4,88 \\
- sem especificar & 3 & $\mathbf{7 , 3 2}$ \\
\hline Subtotal & $\mathbf{5}$ & $\mathbf{1 2 , 2 0}$ \\
\hline Total & $\mathbf{4 1}$ & $\mathbf{1 0 0 , 0 0}$ \\
\hline
\end{tabular}


Para os docentes, as ações assistências aparecem em primeiro lugar com 48,78\% das respostas e os enfermeiros apontam-na em 33,60\% das mesmas, entretanto os respondentes são concordantes em priorizar as ações de prevenção de incapacidade. A educação em saúde foi referida por $39,02 \%$ dos docentes e por $42,46 \%$ dos enfermeiros, sendo que estes últimos ressaltaram a importância das ações envolvendo a socialização do paciente que nesta patologia geralmente é muito comprometida.

TABELA 2 - DISTRIBUIÇÃO DAS RESPOSTAS DOS ENFERMEIROS SOBRE OS ASPECTOS IMPORTANTES DA ATUAÇÃO DO ENFERMEIRO EM HANSENÍASE

\begin{tabular}{|c|c|c|}
\hline \multirow{2}{*}{ Aspectos importantes } & \multicolumn{2}{|c|}{ Respostas } \\
\hline & N. ${ }^{\circ}$ & $\%$ \\
\hline \multicolumn{3}{|l|}{ Educação em saúde } \\
\hline - socialização do paciente & 53 & 8,70 \\
\hline - orientação sobre o tratamento & 44 & 7,21 \\
\hline - orientação sobre a doença & 41 & 6,72 \\
\hline - junto a população & 16 & 2,62 \\
\hline - trabalho de grupo & 13 & 2,13 \\
\hline - autocuidado & 6 & 0,98 \\
\hline - sem especificar & 86 & 14,10 \\
\hline Subtotal & 259 & 42,46 \\
\hline \multicolumn{3}{|l|}{ Assistência de enfermagem } \\
\hline - prevenção de incapacidades & 132 & 21,63 \\
\hline - curativos & 26 & 4,26 \\
\hline - assistência direta & 23 & 3,77 \\
\hline - consulta de enfermagem & 14 & 2,30 \\
\hline - coleta de material para exames/teste & 6 & 0,98 \\
\hline - assistência integral & 2 & 0,33 \\
\hline - exame físico geral & 2 & 0,33 \\
\hline Subtotal & 205 & 33,60 \\
\hline \multicolumn{3}{|l|}{ Vigilância epidemiológica } \\
\hline - controle de comunicantes & 49 & 8,03 \\
\hline - visita domiciliária & 24 & 3,93 \\
\hline - controle de endemia & 14 & 2,30 \\
\hline - sem especificar & 37 & 6,06 \\
\hline Subtotal & 124 & 21,63 \\
\hline \multicolumn{3}{|l|}{ Administração de serviços } \\
\hline - treinamento de pessoal & 11 & 1,80 \\
\hline - organização & 3 & 0,49 \\
\hline - supervisão & 3 & 0,49 \\
\hline - execução & 2 & 0,33 \\
\hline - avaliação & 2 & 0,33 \\
\hline - planejamento & 1 & 0,16 \\
\hline Subtotal & 22 & 3,60 \\
\hline Total & 610 & 100,00 \\
\hline
\end{tabular}


Em relação aos aspectos da atuação do enfermeiro apresentados nas Tabelas 1 e 2 é necessário levar em consideração que a prática de enfermagem, conforme citado por Almeida', não é apenas uma prática técnico-científica que vai produzindo ao longo do tempo um conhecimento sobre o cuidar, ou seja, de como fazê-lo sempre cada vez melhor e de como organizá-lo e administrá-lo mais lógica e racionalmente, isto porque entende-se a prática de enfermagem como prática social e portanto historicamente estruturada e socialmente articulada.

Por outro lado, a enfermagem faz parte de um processo coletivo de trabalho composto de áreas técnicas especificas e, particularmente, dentro do Programa de Controle da Hanseníase, este deve contar com o trabalho de uma equipe multiprofissional, onde devem ser compartilhadas parcelas das diferentes atuações visando compor um conjunto complementar e interdependente como forma de contribuir para a integralidade da assistência a saúde.

A análise histórica dos modelos de intervenção sobre hanseníase aponta para uma mudança muito pouco significativa na situação epidemiológica, ao contrário, o que se tem observado é um aumento de endemia.

A conjuntura atual brasileira na área da saúde e em particular a do Programa de Controle de Hanseníase requer o desenvolvimento de ações e decisões voltadas para um efetivo compromisso com a saúde da população e nesse sentido, constitui oportunidade histórica para o estabelecimento de novas práticas de saúde e com isto a necessidade de repensar a atuação do enfermeiro junto a esse programa.

Vale ressaltar que com a introdução do novo esquema terapêutico, ou seja, a poliquimioterapia ${ }^{2}$ objetiva-se reduzir as fontes de infecção e conseqüentemente, prevenir a hanseníase e, portanto abre-se um espaço de trabalho muito amplo para diferentes profissionais que através do desenvolvimento dessa nova tecnologia vem apresentar uma maior perspectiva na organização e administração dos serviços de saúde.

A ênfase dada na participação dos diferentes profissionais, em particular do enfermeiro, nas várias etapas do processo de trabalho, sugere a necessidade de revisão das práticas desenvolvidas, de modo a adequar as ações realizadas dentro do novo modelo tecnológico às necessidades de saúde da clientela atendida junto ao Programa de Controle da Hanseníase, cabendo, portanto, aos órgãos coordenadores do referido Programa e às instituições formadoras de profissionais da área da saúde, revisar e atualizar os conhecimentos produzidos acerca da hanseníase junto aos serviços de atendimento à clientela que apresenta esse problema de saúde. 


\section{ASSESSMENT OF NURSING ACTIONS IN THE LEPROSY CONTROL PROGRAM IN THE STATE OF SÃO PAULO}

The present study assesses nursing actions in the Leprosy Control Program in the

state of São Paulo. Nursing care, education, epidemiological control and administrative actions are primitive. Revision of practices being developed in the new model of health care is necessary.

UNJTERMS: nursing, practices, leprosy

\section{ESTUDIO SOBRE LAS ACCIONES DE ENFERMERÍA EN EL PROGRAMA DE CONTROLE DE LA LEPRA EN SÃO PAULO}

En este estudio se discute sobre las acciones de enfermería en el Programa de Controle del la Lepra en el Estado de São Paulo destacando como siendo primordiales las acciones asistenciales, educativas, de vigilancia epidemiológica y administrativas; se resalta además la necesidad de revisión de las prácticas desarrolladas en el nuevo modelo de asistencia a la salud.

UNITERMOS: enfermería, prácticas, lepra

\section{REFERÊNCIAS BIBLIOGRÁFICAS}

01. ALMEIDA, M.C.P. et al. A situação da enfermagem nos anos 80: desafios da enfermagem para os anos 90. In: CONGRESSO BRASILEIRO DE ENFERMAGEM, 41, Florianópolis, 02 a 07.09.1989. Florianópolis, 1989. Anais. Florianópolis: ABEN, 1989.

02. BRASIL. Ministério da Saúde. Normas técnicas e procedimentos para utilização dos esquemas de poliquimioterapia no tratamento da hanseníase. Brasília: Ministério da Saúde, 1990. p. 70.

03. ENCONTRO ESTADUAL DE AVALIAÇÃO, 3, São Paulo, 24 a 26.04.1991. São Paulo: Secretaria de Estado da Saúde, 1991. 
04. OFICINA DE TRABALHO DE AÇÕES DE ENFERMAGEM EM HANSENÍASE, 1, Campos de Jordão, 01 a 04.07.1991. São Paulo: Secretaria de Estado da Saúde/Coordenação do Programa de Controle da Hanseníase, 1991.

05. ORGANIZACIÓN PANAMERICANA DE LA SALUD. La participación del personal de enfermería en el control de la lepra: propuesta. Washington, 1989. p. 16. (Informe Final do Grupo de Trabalho - Brasil)

06. PEDRAZZANI, E. S. O ensino e a prática do enfermeiro em hanseníase. São Paulo, p. 139. Tese (Doutorado) - Faculdade de Saúde Pública - Universidade de São Paulo, 1990. 